\title{
Management of Lower Calyceal Stones: Our Experience with Extracorporeal Shock Wave Lithotripsy
}

El Boukili EL Makhoukhi Zayd*, Abdallah Hussein, Dergamoun Hamza, Hachem Al Sayegh, Benslimane Lounis, Nouini Yassine

"A" Department of Urology, Ibn Sina Teaching Hospital

DOI: $10.36347 /$ sasjs.2020.v06i08.003

| Received: 30.05.2020 | Accepted: 08.06.2020 | Published: 09.08.2020

*Corresponding author: El Boukili El Makhoukhi Zayd

Background: The democratization of extracorporeal shock wave lithotripsy (ESWL) in the end of eighties was a worldwide revolution in the management of urolithiasis, thanks to Chaussy and al. nowadays, despite all new technologies, ESWL remain very used and allow clinicians to treat patient in the most non-invasive way. Material and methods: In the period between January 2017 and December 2019, 53 patients with isolated lower polar calyceal (LPC) calculi $\leq 25 \mathrm{~mm}$, in patients aged $\geq 18$ years, were included in the study. Non-enhanced computed tomography or Intravenous urograms were reviewed to define the LPC anatomy (width of the infundibulum and pelvicalyceal angle) and to determine the size of stones. Only patients with radiopaque stones were included. The stone-free rates were assessed with plain X-ray and ultrasound at 4 weeks. Result: The average age of the patients was 42 years with extremes ranging from 18 to 70 years. A male predominance is noted with a Sex Ratio (M / F) of 1.3. Among the 53 patients treated with ESWL, an overall rate of no fragment (Stone Free) was obtained in 55\% of our patients. There were no major complications to report during or after ESWL sessions.

Keywords: Extracorporeal lithotripsy, kidney stones, lower caliceal group.

Copyright @ 2020: This is an open-access article distributed under the terms of the Creative Commons Attribution license which permits unrestricted use, distribution, and reproduction in any medium for non-commercial use (NonCommercial, or CC-BY-NC) provided the original author and source are credited.

\section{INTRODUCTION}

The use of acoustic shock waves in the treatment of urinary stones was first made in humans in Germany in 1982. Since then extracorporeal lithotripsy has spread around the world as a revolutionary therapeutic means in the management of urolithiasis of the upper urinary tract.

Over the past twenty years, the ESWL has been perfected and evaluated in numerous studies published in the literature. The results are sometimes controversial. However, situations persist where the choice of the technique to be used in first-line treatment can prove difficult. This is the case of stones in a specific anatomical situation or those associated with an abnormality of the excretory pathway. Lower calyceal stones still pose a problem of optimal care today.

The aim of this work was to evaluate the effectiveness of ESWL in the treatment of stones in the lower calyceal group in the urology department A at Ibn Sina RABAT hospital.

\section{MATERIEL AND METHODS}

This was a retrospective study done over a period of 3 years from January 2017 to December 2019. The radiological and clinical data of all adult patients who were treated for isolated, solitary, radiopaque lower-pole renal stones between less than $25 \mathrm{~mm}$ in the largest dimension by either ESWL were included in the study

All our patients in were treated as outpatients by use of a Siemens Modularis lithotriptor under oral analgesia by using non-steroidal anti-inflammatory (NSAIDs). The decision for pre-ESWL stent placement was according to the preference and decision of the treating consultants; no definite protocol was followed. All patients were treated by two operators under the supervision of an admitting consultant. The rate of administration of shock waves was $60-90 / \mathrm{min}$. All cases were followed up weekly with X-ray and ultrasound to assess fragmentation and clearance. Follow-up SWL sessions were planned at least 1 week after the prior session. SWL was considered to have failed if no fragmentation was noted after 3 sessions. 
All patients were followed up 4 weeks after the last session of ESWL. The stone-free rate was defined as no evidence of stone fragments on a plain X-ray or ultrasound during follow-up. Clinically insignificant residual fragments were defined as stone fragments of 3 $\mathrm{mm}$ or less in largest dimension without any symptoms (pain, fever, hematuria, etc.) or evidence of obstruction

\section{RESULTS}

A total of 632 patients underwent ESWL for renal calculi during the study period, of whom 53 $(8,3 \%)$ fulfilled the inclusion criteria and were included in the study.

We conducted a retrospective study of 53 patients with 56 kidney stones in the lower calyceal group who were treated with extra-bodily lithotripsy in the "A" urology department at Ibn-Sina hospital in Rabat during the period between January 2017 and December 2019

The average age of the patients was 42 years with extremes ranging from 18 to 70 years. These were 30 men $(57 \%)$ and 23 women $(43 \%)$. There is a clear male predominance with a sex ratio of 1.3 .

The predominant reason for consultation was lumbagos $(60 \%)$, followed by hematuria $(10 \%)$ then urinary tract infection $(5 \%)$. The remaining cases were asymptomatic and were discovered fortuitously during non-oriented assessments for urinary symptomatology.

$30 \%$ of the patients treated had residual lithiasis not eliminated after a percutaneous nephrolithotomy, nor surgical pyelonephrolithotomy.

All the stones of the lower calyceal system, measured $25 \mathrm{~mm}$ or less. 3 patients were carriers of multiple lithiasis $(5.7 \%)$.

The lithiasis was unilateral on the left in 26 patients (49\%), on the right in 24 patients $(45.3 \%)$ and bilateral in 3 patients $(5.7 \%)$.

The diameter of the lithiasis or the lithiasic residue to be treated was measured in all our patients. The average diameter at the time of treatment was 14 $\mathrm{mm}$ (range: 6 and $25 \mathrm{~mm}$ ).

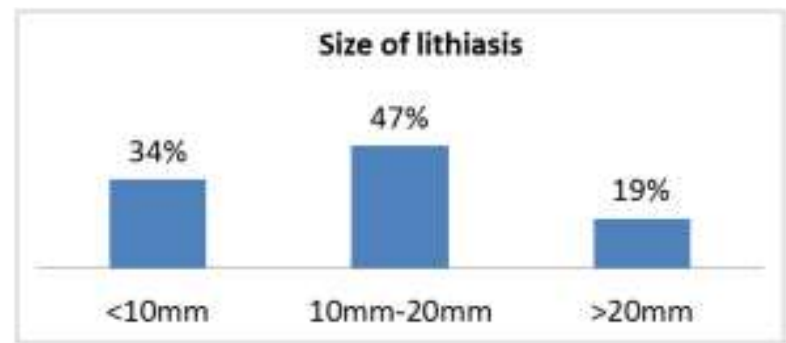

Fig-1: The distribution of lithiasis or residues according to their diameter at the time of treatment
The chemical nature of lithiasis was determined in 30 patients $(56 \%)$. The distribution based on the predominant-chemical compound is presented in Table 1.

Table-1: Distribution according to the result of the spectrophotometric study of the calculations Phospho-calcium lithiasis 7 patients

\begin{tabular}{|l|l|}
\hline Oxalo-calcium lithiasis & 22 patients \\
\hline Uric lithiasis
\end{tabular}

\begin{tabular}{|l|l|}
\hline Uric lithiasis & 1 patient
\end{tabular}

The total number of stones treated with LEC is 56 stones in 53 patients.

The size of the stones treated by ESWL was less than $10 \mathrm{~mm}$ in 18 cases, between 10 and $20 \mathrm{~mm}$ in 25 cases and more than $20 \mathrm{~mm}$ in 10 cases.

29 of our patients or $55 \%$ had no fragment (Stone Free) after 3 LEC sessions; in the other side 24 patients $(45 \%)$ presented a failure by persistence of residual fragments despite 3 LEC sessions. (Table 2) $.77 \%$ of stones (14/18) measuring $10 \mathrm{~mm}$ or more were correctly fragmented after ESWL, compared to $43 \%$ $(15 / 35)$ of stones measuring more than $10 \mathrm{~mm}$. (Table $3)$.

Lithotripsy hadn't resulted in any noticeable disorder justifying its interruption.

Most of our patients presented with transient hematuria, self-limiting, along with often moderate pain which corresponds with the stones' migration to the lower urinary apparatus.

Table-2: Overall results in our study

\begin{tabular}{|l|l|}
\hline & Results \\
\hline Success & $55 \%$ \\
\hline Failure & $45 \%$ \\
\hline
\end{tabular}

Table-3: Results based on the size of the stones

\begin{tabular}{|l|l|l|}
\hline Size & Success & Failure \\
\hline Stone $<10 \mathrm{~mm}$ & 14 cases & 4 cases \\
\hline Stone $>10 \mathrm{~mm}$ & 15 cases & 20 cases \\
\hline
\end{tabular}

\section{DISCUSSION}

The results of the ESWL depend on the stone (size, nature, location), of the patient (age, BMI), but also on other parameters (lithotripter, technical operator).

Upper and middle calyceal stones smaller than $15 \mathrm{~mm}$ are preferential and often processed by ESWL. The same is not true for lower calyceal stones [1].

In case of lower calice localization, it is rather the elimination than the fragmentation of the stones after ESWL that is called into question. In general, the rate of Fragment Free (Stone Free) for the treatment of stones of the lower calice by ESWL is estimated at $63 \%$ (55\% in our series) while it is $73,69,80$ and $88 \%$ 
respectively for the upper calice, the middle calice, the pyelon and the pyelo-ureteral junction.

On a meta-analysis of 2927 patients treated by ESWL, Lingeman and al. have shown that the rate of Stone Free was directly linked to the location and size of the stones [2].

The Stone free rate was $74 \%$ for stones less than $10 \mathrm{~mm}$ and $56.3 \%$ for stones from 10 to $20 \mathrm{~mm}$. ESWL is strongly recommended as a first-line treatment for the treatment of symptomatic lower calyceal stones lower than $10 \mathrm{~mm}$. The situation is less clear for stones lower than 10 to $20 \mathrm{~mm}$ in diameter, which was the case in our series.

These poor results in terms of the lower calyceal stones can be explained by the declining position of the latter and by the particular anatomical arrangement of the lower pole of the kidney and a long and / or narrow calyceal rod [3].

Sampaio and al. reported a Stone Free (SF) rate of $75 \%$ after ESWL for calyceal stones less than 7 to $25 \mathrm{~mm}$ in diameter when the infundibulo-pelvic angle was greater than $90^{\circ}$. Conversely, in the case of a more acute angle, less than $90^{\circ}$, the rate of Stone Free was only $23 \%$ [4].

Gupta and al. also reported a correlation between the infundibulo-pyelic angle and the rate of Stone Free after LEC, confirming the work of Sampaio and al. They also demonstrated that the rate of Stone Free increases when the lower calyceal system measures less than $3 \mathrm{~cm} \mathrm{[5].}$

In our series, there was not much difference between patients with different sized calice systems. Graff et al. obtained Stone Free rates of $81 \%$ and $83 \%$, respectively, in case of uric acid or calcium oxalate dihydrate stones $[6,7]$. It is not the same in case of stones of cystine, brushite or calcium oxalate monohydrate. Several authors do not recommend ESWL as a first-line treatment for this type of calculation if their size exceeds $10 \mathrm{~mm}$, particularly in a lower calyceal situation $[6,8,9]$. This parameter was not taken into account in our study.

Regarding postural therapy to promote the elimination of lower calyceal fragments. In 1990, Brownlee and al. reported their experience with a postural therapy protocol (patient in head-up position) associated with percussion of the lumbar fossa and diuresis treatment to facilitate the evacuation of residual fragments after LEC. After two weeks of such treatment, the rate of FS was $88 \%$ compared to $12.5 \%$ for the untreated group [10].

Pace and al. also have demonstrated in a prospective randomized study the interest of postural therapy associated with a course of diuresis and a percussion of the lumbar fossa with a rate of SF of $40 \%$ against $3 \%$ for the control group [11]. Finally, these data were recently confirmed by Chiong et al. [12].

This measure was not undertaken in our study.

\section{REFFERENCE}

1. Pearle MS, Traxer O. Renal urolithiasis: surgical therapy for special circumstances; 2001. AUA Update Series 2001

2. Lingeman JE, Siegel YI, Steele B. Management of lower pole nephrolithiasis: critical analysis. J Urol.1994;151:663-7.

3. Havel D, Saussine C, Fath C, Lang H, Faure F, Jacqmin D. Singlestones of the lower pole of the kidney.Comparative results of extracorporeal shock wave lithotripsy and percutaneous nephrolithotomy. EuropeanUrology.1998;33:396-400.

4. Sampaio FJ, D’Anunciacao AL, Silva EC. Comparative follow-up of patientswithacuteandobtuseinfundibulumpelvicanglesubmitted toextracorporeal shockwave lithotripsy for lower caliceal stones: preliminaryreportandproposedstudydesign. JEndourol. 1997;11:157-61.

5. Gupta NP, Singh DV, Hemal AK, MANDAL S. Infundibulopelvic anatomy and clearance of inferior caliceal calculi with shock wave lithotripsy. The Journal of urology. $2000 \mathrm{Jan} ; 163(1): 24-7$.

6. GraffJ, Diederichs W, Schulze H. Longtermfollowupin1003extracor- poreal shock wave lithotripsy patients. J Urol1988;140:479-83

7. Newman DM, Scott JW, Lingeman JE. Two-year follow-up of patients treated with extracorporeal shock wave lithotripsy. Journal of Endourology. 1988;2(2):163-71.

8. Klee LW, Brito CG, Lingeman JE. Theclinicalimplicationsofbrushite calculi. J Urol.1991;145:715-8.

9. Hockley NM, Lingeman JE, Hutchinson CL. Relative efficacy of extracorporeal shock wave lithotripsy and percutaneous nephrostolithotomy in the management of cystine calculi. Journal of Endourology. 1989;3(3):27385.

10.Brownlee N, Foster M, Griffith DP, Carlton CE. Controlled inversion therapy: an adjunct to the elimination of gravity-dependent fragments following extracorporeal shock wave lithotripsy. The Journal of urology. 1990 Jun;143(6):1096-8.

11.Pace KT, Tariq N, Dyer SJ, Weir MJ, D'a Honey RJ. Mechanical percussion, inversion and diuresis for residual lower pole fragments after shock wave lithotripsy: a prospective, single blind, randomized controlled trial. J Urol. 2001;166:2065-71.

12. Chiong E, Hwee ST, Kay LM, Liang S, Kamaraj R, Esuvaranathan K. Randomized controlled study of mechanical percussion, diuresis, andinversiontherapytoassistpassageoflowerpolerenalcal culiafter shock wave lithotripsy. Urology. 2005;65:1070-4. 